\title{
Exploring the Potential Pharmacologic Mechanism of Heterophyllin B in the Treatment of Esophageal Cancer by Network Pharmacology
}

\author{
Ting Chen ${ }^{2}$, Liang Zhang ${ }^{1}$, Xulong Huang ${ }^{1}$, Haiyu Chen ${ }^{1}$, Shengpeng Zhu ${ }^{1}$, Chao Huang ${ }^{2}$, Bin Huang,"* \\ ${ }^{1}$ School of Pharmaceutical Sciences, Hunan University of Medicine, Huaihua 418000, China \\ ${ }^{2}$ Department of Pharmacy, Guizhou Health Vocational College, Tongren 554301, China
}

\begin{abstract}
This study used the method of network pharmacology to preliminarily predict the mechanism of Heterophyllin $\mathrm{B}(\mathrm{HB})$ inhibiting Esophageal Cancer(EC). We found the HB targets in the TCMSP and PuChem databases, and searched all EC-related targets in the GeneCards database. Taken the intersection of $\mathrm{HB}$ and EC as potential targets for inhibiting EC, and used Cytoscape 3.7.1 software to perform topological analysis on potential targets to obtain core targets. Used the start Analysisi function in the DAVID database to analyzed the biological process of the core target, and visualized it with the the R language tool. As a result, 75 potential targets for inhibiting EC were obtained, of which MMP9, MMP2, CCND1, STAT3, CXCR4, BDKRB1and PTGS2 were the main core targets. HB inhibits the occurrence of EC through Pathways in cancer, TNF signaling pathway, Bladder cancer, Small cell lung cancer, Rheumatoid arthritis related pathways, mainly involving proteolysis, collagen catabolic process, extracellular matrix disassembly, positive regulation of cell proliferation, positive regulation of cytosolic calcium ion concentration biological processes. This study initially revealed the molecular mechanism of HB inhibiting EC, and provided a reference for HB to expand new indications.
\end{abstract}

\section{Introduction}

Esophageal Cancer ( EC) is a common malignant tumor of the digestive tract, with a high mortality rate, among which men are higher than women[1], and its morbidity and mortality have been on the rise in recent years[2]. When carcinogens or external mechanical forces continuously stimulate the esophageal mucosa, EC will continue to develop[3-4]. The treatment of EC is still based on traditional radiotherapy and chemotherapy, and there is no particularly effective treatment for EC, which has also led to a continuous decline in the survival rate of patients[5]. In recent years, the development of anti-tumor drugs from Traditional Chinese Medicine

(TCM) is a hot topic. Of course, TCM has also made significant contributions in the process of human antitumor[6].

Heterophyllin B ( HB ) is an important active substance in the plant Pseudostellaria heterophylla (Miq.) Pax et Hoffm. Studies have shown that HB has antiinflammatory, anti-tumor, anti-fungal, and anti-bacterial activities[7-10]. HB has a certain inhibitory effect on a variety of tumor cells, but there are few reports on the effect of $\mathrm{HB}$ on $\mathrm{EC}$, and its mechanism of action has not been elucidated.

Based on the analysis methods of network pharmacology and molecular informatics, this study initially discussed the molecular mechanism of $\mathrm{HB}$ inhibiting EC, and provided reference for the clinical application of $\mathrm{HB}$.

\section{Materials and methods}

\subsection{HB-related targets}

We collect the targets of $\mathrm{HB}$ and establish the active ingredient target data set through TCMSP and Swiss TargetPrediction(http://www.swisstargetprediction. $\mathrm{ch} /$ ) database.

\subsection{EC-related targets}

With "Esophageal Cancer" as a key word, We search the EC-related targets and establish disease targets data set through the GeneCards database(https://www.genecards. org/).

\subsection{Network construction}

We use the intersection of drugs and disease targets to find potential targets for $\mathrm{HB}$ to inhibit EC. The PPI network of potential targets was constructed through the String database (https://string-db.org/), cytoscape 3.7.1 software visually analyzed the PPI network . In this way,

\footnotetext{
* Corresponding author: huangbinsg@163.com
} 
the median values of "Degree", "Betweenness centrality" and "Closeness centrality" are calculated.

\subsection{Bioinformatic analysis}

Genetic ontology (GO) analysis of biological processes and Kyoto Encyclopedia of Genes and Genomes (KEGG) enrichment analysis through the DAVID (https://david. ncifcrf.gov/tools.jsp). The $\mathrm{R}$ language tool performs visual analysis, $\mathrm{P} \leq 0.05$ as the screening criteria to screen out biological processes, cell compositions, molecular functions and signal pathways with extremely significant differences.

\subsection{Pathway mapper construction}

The core targets are mapped to related pathways mainly through KEGG (https://www.genome.jp/kegg/)Mapper function.

\section{Results}

\subsection{Target prediction}

We obtained 108 targets corresponding to HB in TCMSP and Swiss Target Prediction database, and 5801 EC targets were obtained in GeneCards database.The results are shown in Figure 1.

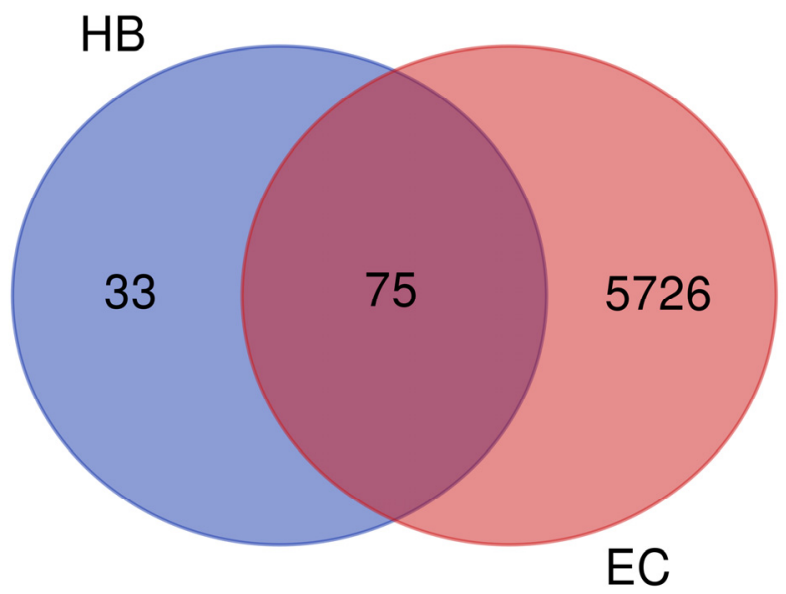

Fig. 1. Drug-disease intersection target Venn diagram

\subsection{Screening of core targets}

Cytoscape 3.7.1 software was used for visual analysis, and the core target was selected that satisfies the median value of more than three topological parameters at the same time. The median values of the three topological parameters are 11 (Degree), 0.0070 (Betweenness centrality), 0.4534 (Closeness centrality). The results are shown in Table 1 and Figure 3.

\subsection{Drug-disease-core target network constru- ction}

Cytoscape 3.7.1 software was used constructed "drugdisease-core target" network, and the results are shown in Figure 2.

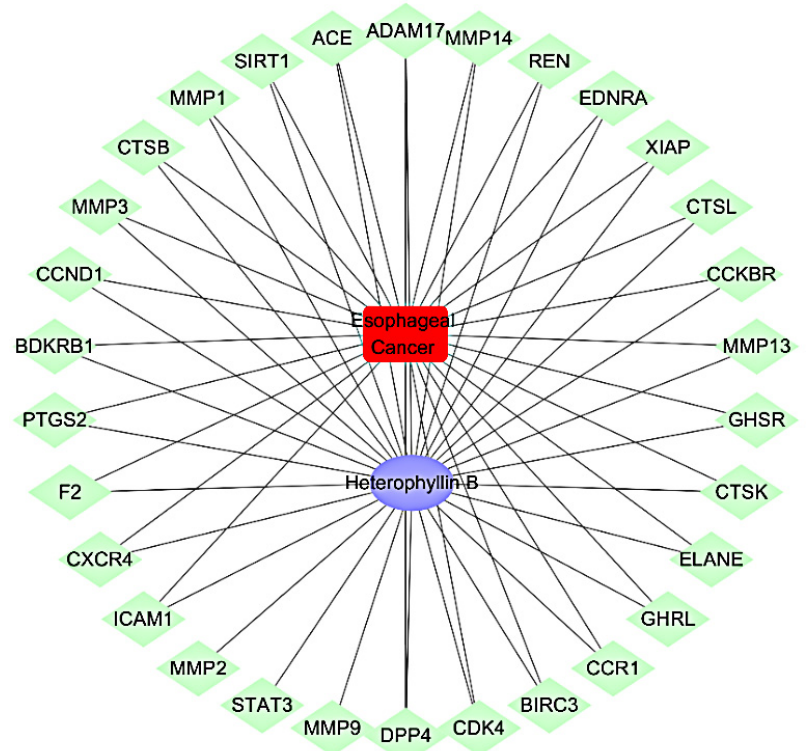

Fig.2. Drugs-diseases-core targets network

\subsection{GO Biological process enrichment analysis}

The top 10 biological processes, cell composition and molecular functions were selected from 86 biological processes. The results are shown in Figure 4. The results show that HB may inhibit EC through multiple biological processes such as proteolysis, collagen catabolic process, extracellular matrix disassembly, positive regulation of cell proliferation, positive regulation of cytosolic calcium ion concentration.

Table 1. Related topological parameters of the core target

\begin{tabular}{|c|c|c|c|c|c|}
\hline UniProt CID & Gene name & Protein name & Degree & $\begin{array}{l}\text { Closeness } \\
\text { Centrality }\end{array}$ & $\begin{array}{l}\text { Betweenness } \\
\text { Centrality }\end{array}$ \\
\hline P14780 & MMP9 & Matrix Metallopeptidase 9 & 35 & 0.6460 & 0.1087 \\
\hline P40763 & STAT3 & $\begin{array}{l}\text { Signal Transducer And Activator Of } \\
\text { Transcription } 3\end{array}$ & 29 & 0.6134 & 0.1304 \\
\hline P08253 & MMP2 & Matrix Metallopeptidase 2 & 27 & 0.6033 & 0.0536 \\
\hline P05362 & ICAM1 & Intercellular Adhesion Molecule 1 & 26 & 0.5935 & 0.0852 \\
\hline P61073 & CXCR4 & C-X-C Motif Chemokine Receptor 4 & 25 & 0.5794 & 0.0882 \\
\hline P00734 & $\mathrm{F} 2$ & Coagulation Factor II, Thrombin & 24 & 0.5573 & 0.0988 \\
\hline P35354 & PTGS2 & Prostaglandin-Endoperoxide Synthase 2 & 23 & 0.5703 & 0.0459 \\
\hline
\end{tabular}




\begin{tabular}{|c|c|c|c|c|c|}
\hline P46663 & BDKRB1 & Bradykinin Receptor B1 & 20 & 0.4679 & 0.0366 \\
\hline P24385 & CCND1 & Cyclin D1 & 20 & 0.5368 & 0.0468 \\
\hline P08254 & MMP3 & Matrix Metallopeptidase 3 & 18 & 0.5252 & 0.0097 \\
\hline P07858 & CTSB & Cathepsin B & 18 & 0.4679 & 0.0468 \\
\hline P03956 & MMP1 & Matrix Metallopeptidase 1 & 17 & 0.5214 & 0.0114 \\
\hline Q96EB6 & SIRT1 & Sirtuin 1 & 17 & 0.5177 & 0.0432 \\
\hline P12821 & $\mathrm{ACE}$ & Angiotensin I Converting Enzyme & 16 & 0.5105 & 0.0131 \\
\hline P78536 & ADAM17 & ADAM Metallopeptidase Domain 17 & 16 & 0.5105 & 0.0156 \\
\hline P50281 & MMP14 & Matrix Metallopeptidase 14 & 16 & 0.5368 & 0.0237 \\
\hline P00797 & REN & Renin & 15 & 0.5328 & 0.0290 \\
\hline P25101 & EDNRA & Endothelin Receptor Type A & 15 & 0.4740 & 0.0169 \\
\hline P98170 & XIAP & X-Linked Inhibitor Of Apoptosis & 15 & 0.4867 & 0.0162 \\
\hline P07711 & CTSL & Cathepsin L & 15 & 0.4534 & 0.0267 \\
\hline P32239 & CCKBR & Cholecystokinin B Receptor & 14 & 0.4534 & 0.0093 \\
\hline P45452 & MMP13 & Matrix Metallopeptidase 13 & 14 & 0.5177 & 0.0100 \\
\hline Q92847 & GHSR & Growth Hormone Secretagogue Receptor & 14 & 0.4620 & 0.0202 \\
\hline P43235 & CTSK & Cathepsin K & 14 & 0.4740 & 0.0087 \\
\hline P08246 & ELANE & Elastase, Neutrophil Expressed & 14 & 0.5034 & 0.0146 \\
\hline Q9UBU3 & GHRL & Ghrelin And Obestatin Prepropeptide & 13 & 0.4834 & 0.0299 \\
\hline P32246 & CCR1 & C-C Motif Chemokine Receptor 1 & 12 & 0.5141 & 0.0193 \\
\hline Q13489 & BIRC3 & Baculoviral IAP Repeat Containing 3 & 12 & 0.4834 & 0.0073 \\
\hline P11802 & CDK4 & Cyclin Dependent Kinase 4 & 12 & 0.4740 & 0.0174 \\
\hline P27487 & DPP4 & Dipeptidyl Peptidase 4 & 11 & 0.4932 & 0.0107 \\
\hline
\end{tabular}

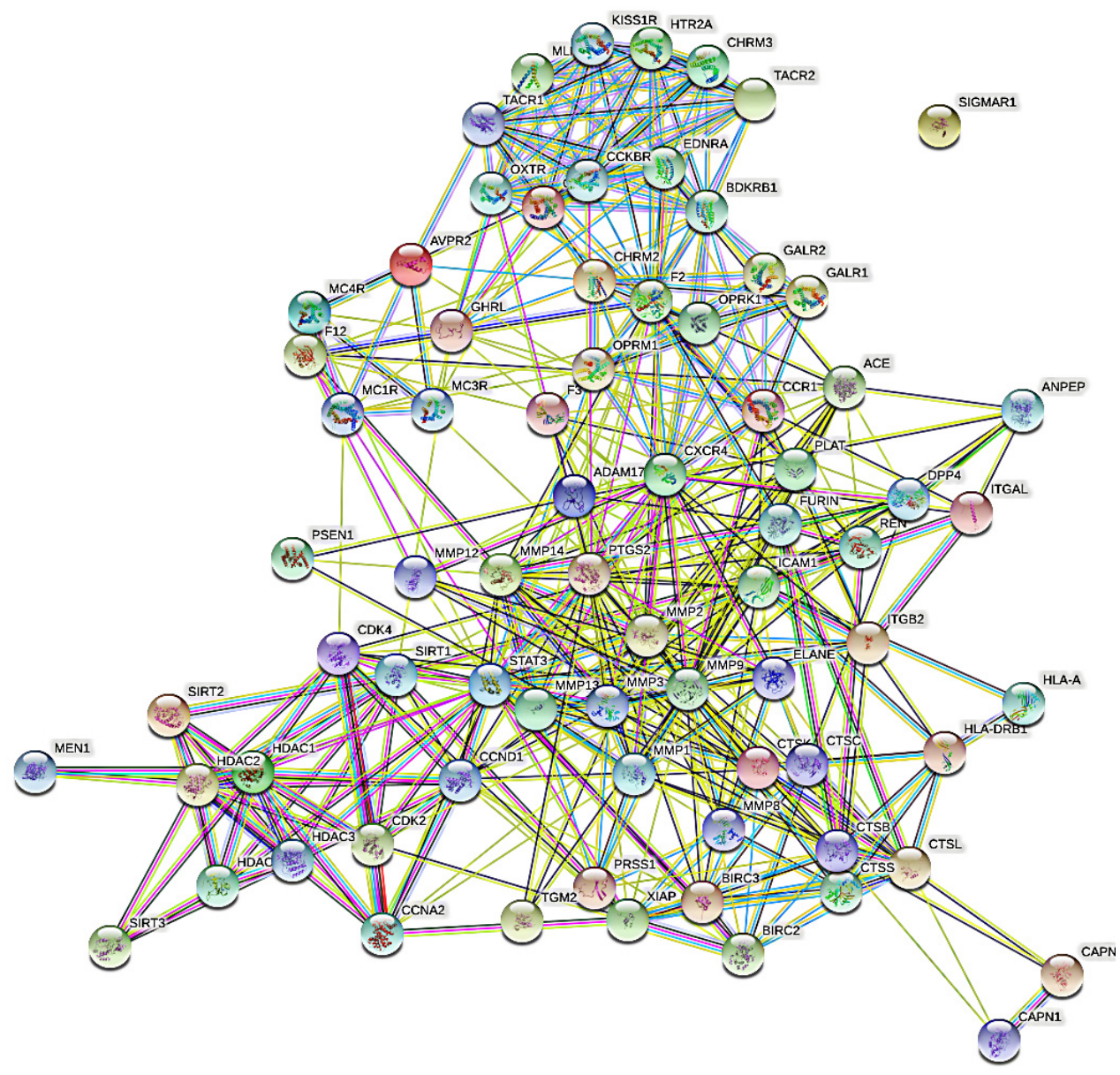

Fig. 3. The protein interaction network of drug-disease intersection target 


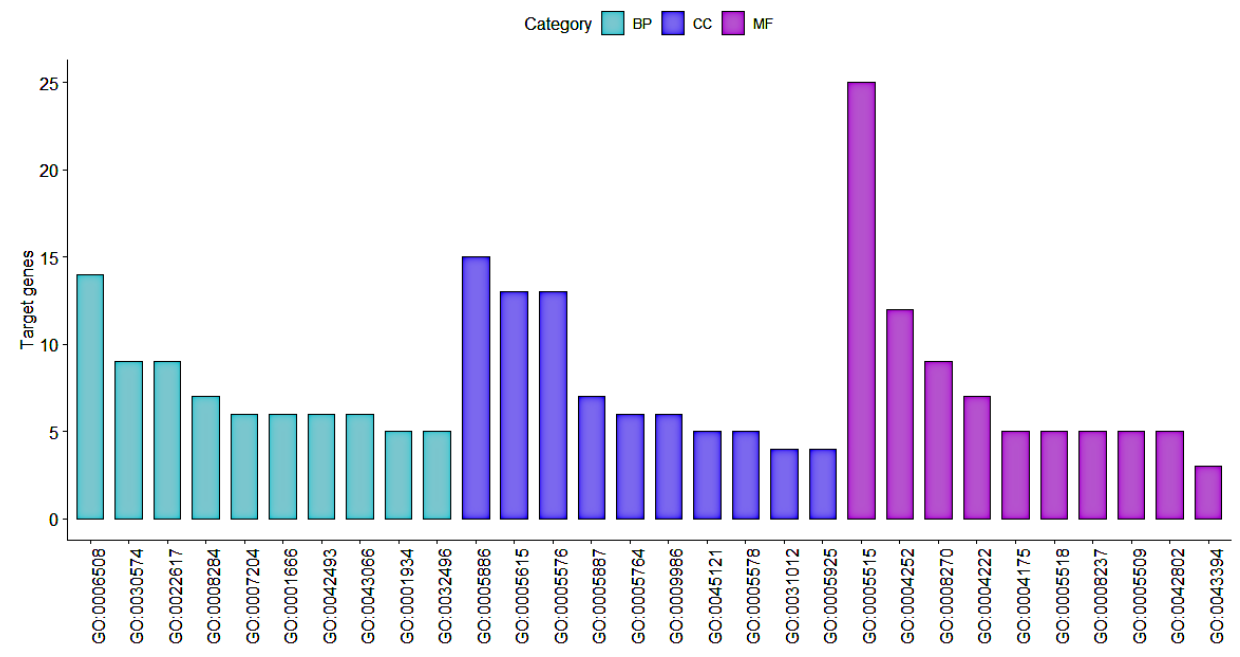

Fig. 4. Top 10 biological process enrichment results

\subsection{KEGG Pathway enrichment analysis}

The 13 signal pathways were enriched and analyzed by KEGG, and the results are shown in Figure 5. The main signaling pathways include Pathways in cancer, TNF signaling pathway, Bladder cancer, Small cell lung cancer, Rheumatoid arthritis related pathways.

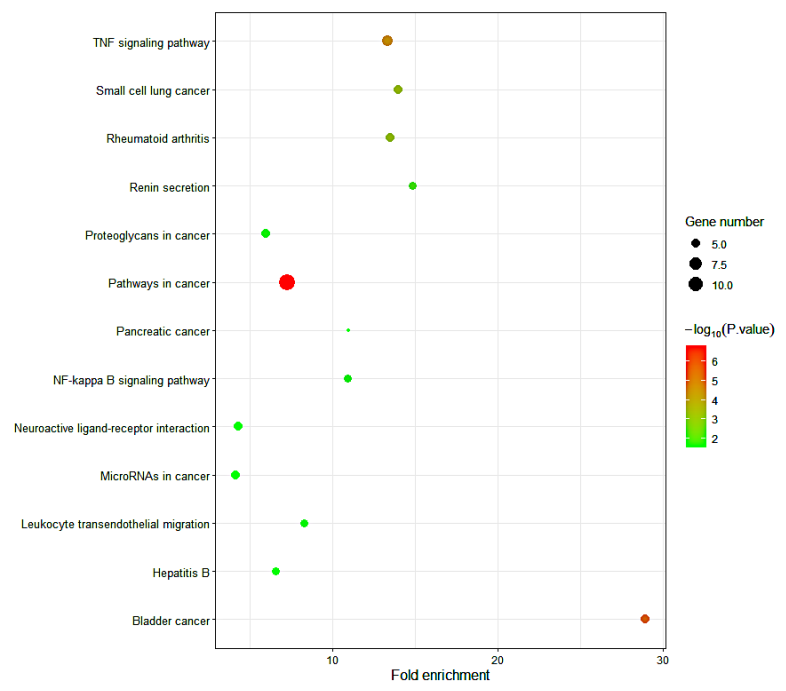

Fig. 5. The 13 pathways enriched by major hubs

\subsection{Pathway annotation diagram of HB's anti-EC effect}

We input the intersection target and mark the number of targets in each pathway through the KEGG Mapper function of the KEGG database. 12 target proteins are involved in the Pathways in cancer.

\section{Discussion}

Through the analysis of network pharmacology, MMP9, MMP2, CCND1, STAT3, CXCR4, BDKRB1, PTGS2, may be the potential targets for HB in the treatment of EC. In the expression of MMPs-related genes, MMP9 and MMP2 are closely related to the metastasis of a variety of human carcinoma, including esophageal cancer[11-12].

GO biological process enrichment analysis demonstrates that HB's anti-EC effect involves biological processes such as collagen catabolic process, extracellular matrix disassembly, proteolysis, positive regulation of cytosolic calcium ion concentration. The main biological process may include proteolysis, collagen catabolic process, extracellular matrix disassembly, positive regulation of cell proliferation, positive regulation of cytosolic calcium ion concentration.

KEGG pathway enrichment analysis showed that HB anti-EC effects through regulating Pathways in cancer, TNF signaling pathway, Bladder cancer, Small cell lung cancer, Rheumatoid arthritis related pathways. The expression of MMP2 and MMP9 levels is mediated by multiple signals, PI3K/AKT is one of the main pathways[13]. HB inhibits the adhesion and invasion of ECA-109 cells mainly through the PI3K/AKT/ $\beta$-catenin pathway that down-regulating the expression of MMP2 and MMP9[14].

\section{Conclusions}

A total of 75 potential targets were obtained, 30 of which are core targets for $\mathrm{HB}$ to inhibit EC. GO biological process enrichment analysis and KEGG pathway enrichment analysis revealed 10 biological processes, cell composition, molecular functions and 13 pathways are closely related to the occurrence and development of inhibiting EC, mainly involving Pathways in cancer, TNF signaling pathway, Bladder cancer, Small cell lung cancer, Rheumatoid arthritis. This study preliminarily explored the mechanism of $\mathrm{HB}$ inhibiting $\mathrm{EC}$ at the molecular level, and provided a reference for the discovery of new targets for EC. 


\section{Acknowledgments}

The authors would like to thank the financial support from the Scientific Research Foundation of Hunan Provincial Education Department (20B415), the Technology Project Foundation of Tongren ([2018]48), the Technology Project Foundation of Tongren ([2019]55).

\section{References}

1. Chen, W., Zheng, R., Baade, P.D., Zhang, S., Zeng, H., Bray, F., Jemal, A., Yu, X.Q., He, J. (2016) Cancer statistics in China, 2015. J. CA Cancer J Clin., 66: 115-32.

2. Torre, L. A., Bray, F., Siegel, R. L., Ferlay, J., Lortet-Tieulent, J., Jemal, A. (2015) Global cancer statistics, 2012. J. CA Cancer J Clin., 65: 87-108.

3. Abnet, C.C., Arnold, M., Wei, W.Q. (2018) Epidemiology of Esophageal Squamous Cell Carcinoma. J. Gastroenterology., 154: 360-373.

4. Smyth, E.C., Lagergren, J., Fitzgerald, R.C., Lordick, F., Shah, M.A., Lagergren, P., Cunningham, D. (2017) Oesophageal cancer. J. Nat Rev Dis Primers., 27: 17048.

5. Wu, C., Huang, H., Choi, H.Y., Ma, Y., Zhou, T., Peng, Y., Pang, K., Shu, G., Yang, X. (2021) Antiesophageal Cancer Effect of Corilagin Extracted from Phmllanthi Fructus via the Mitochondrial and Endoplasmic Reticulum Stress Pathways. J. J Ethnopharmacol., 269: 113700.

6. Anwanwan, D., Singh, S.K., Singh, S., Saikam, V., Singh, R. (2020) Challenges in liver cancer and possible treatment approaches. J. Biochim Biophys Acta Rev Cancer., 1873: 188314.

7. Yang, C., You, L., Yin, X., Liu, Y., Leng, X., Wang, W., Sai, N., Ni, J. (2018) Heterophyllin B Ameliorates Lipopolysaccharide-Induced Inflammation and Oxidative Stress in RAW 264.7 Macrophages by Suppressing the PI3K/Akt Pathways. J. Molecules., 23: 717.

8. Zhao, S.M., Kuang, B., Peng, W.W., He, W.J., Xu, H.M., Ji, C.J., Han, J., Zheng, Y.Q., Song, W.W., Tan, N.H. (2012). Chemical Progress in Cyclopeptide-containing Traditional Medicines Cited in Chinese Pharmacopoeia. J. Chinese J Chem., 30: 1213-1223.

9. Schneider, T., Müller, A., Miess, H., Gross, H. (2014). Cyclic lipopeptides as antibacterial agentspotent antibiotic activity mediated by intriguing mode of actions. J. Int J Med Microbiol., 304: 37-43.

10. Yang, Y.B., Tan, N.H., Zhang, F., Lu, Y.Q., He, M., Zhou, J. (2003) Cyclopeptides and amides from Pseudostellaria heterophylla (Caryophyllaceae). J. Helv Chim Acta., 86: 3376-3379.

11. Samantaray, S., Sharma, R., Chattopadhyaya, T.K., Gupta, S.D., Ralhan, R. (2004) Increased expression of MMP-2 and MMP-9 in esophageal squamous cell carcinoma. J. J Cancer Res Clin Oncol., 130: 37-44.
12. Li, Y., Ma, J., Guo, Q., Duan, F., Tang, F., Zheng, P., Zhao, Z., Lu, G. (2009) Overexpression of MMP-2 and MMP-9 in esophageal squamous cell carcinoma. J. Dis Esophagus., 22: 664-7.

13. Kang, M.H., Oh, S.C., Lee, H.J., Kang, H.N., Kim, J.L., Kim, J.S., Yoo, Y.A. (2011) Metastatic function of BMP-2 in gastric cancer cells: the role of PI3K/AKT, MAPK, the NF- $\kappa$ B pathway, and MMP9 expression. J. Exp Cell Res., 317: 1746-62.

14. Tantai, J.C., Zhang, Y., Zhao, H. (2016) Heterophyllin B inhibits the adhesion and invasion of ECA-109 human esophageal carcinoma cells by targeting PI3K/AKT/ $\beta$-catenin signaling. J. Mol Med Rep., 13: 1097-104. 